\title{
An Empirical Study: CDA-based Data Innovation-driven Intelligent Traffic Control System Model of Canton City
}

\author{
Lu-xia $\mathrm{YI}^{1, \mathrm{a}}$, Yu-cong YO ${ }^{2, b}$ \\ ${ }^{1}$ Guangzhou College of Technology and Business \\ Department of Economic and Trade, China \\ ${ }^{2}$ Guangzhou College of Technology and Business \\ Department of Economic and Trade, China \\ astoneyyc@163.com, ${ }^{\mathrm{b}}$ 61070262@qq.com
}

\begin{abstract}
Keywords: Intelligent Traffic Control; Data Acquire; Innovation-driven; Canton mega-city; Abstract: At present, with the growth of private cars and autos ownership, the traffic flow has soared and become a burden for the development of Canton mega-city in China. The sky-rocket number of autos, especially those private ones, has made traffic congestion and car accidents a significant problem Although people are eager to know the real-time road conditions, this is not supported by traditional traffic control system and thus Intelligent Traffic Control System cries out to address these issues Intelligent Traffic Control System serves as the frontier research subject in China and capturing concerns from both the academic field and society for the meantime data Innovation has witnessed the significant and remarkable changes in Information technology. This paper designs a brand new Intelligent Traffic Control System Model based on CDA and Data Innovation-driven; the main functions of the system consist of data acquirement, data analysis and dada driven process in both macro and micro layers. An Empirical Study has followed up to check the effects of this model by the case study of Canton City in transportation system. A positive statistical result has obtained, confirming the positive effect of this model. The results have shown a high practicability and reliability in practical use. With the data acquired, collected and innovatively-driven by monitors installed and decoded in intersections and sub-sections, different road conditions are discriminated, calculated and shown in diverse modes on the controlling layer to allocate efficient paths/routes to avoid the traffic congestion; meanwhile this model renders a guideline for a traffic authority to make decisions on traffic control.
\end{abstract}

\section{Introduction}

Canton city, the center city of Canton province, thanks to the economic progress and regional economic acceleration, Canton mega-city has become one of the most prosperous and developed region in South China. In light of sky-rocketing demand of traffic stimulated by fast development of private cars and autos ownership , traffic flow requirements of traffic control and safety management has been required urgently. By means of lifting the level of intelligent traffic control system, perilous events like traffic accidents can be lowered, and thus traffic safety increased; Some other traffic dilemma can be solved, such as traffic congestion, leading to traffic benefit gained or improved; simultaneously, intelligent traffic control system functions as well to reduce the pollution and contamination of the local and regional environment, especially by means of reducing energy consumption to achieve the low-carbon effect. Quite a number of countries and regions in the world wide scope have paid special heeds on CDA-based Data Innovation-driven, a research hotspot of information technology, which has been applied widely. Intelligent Traffic Control System serves as an extensive, broad, and comprehensive of numerous high-tech fields and domains of research. The design of this system can be given as follows. 


\section{CDA-based Data innovation-driven}

\section{Definition}

CDA-based Data innovation-driven consists of three basic elements. CDA is an abbreviation for Comprehensive Data Acquirement in that Data acquirement process and procedures are carried out from comprehensive aspects. Data innovation is coined as the one of the innovative mechanism in a data collection, acquirement and analysis manners. Innovation-driven in the data layer is thus defined as a driving force stemming from data innovative conduct.

\section{Architecture}

\section{Reality and VR link functions}

It becomes the bridge between the physical outside world and digital VR (virtual reality). It boasts the edges and advantages, such as instant distance, diverse objectives, and high speed and so on and so forth.

\section{RFID RW tag}

RFID RW tag, virtually an emitting and receiving device equipped with antenna, reading and writing, boasts its huge computing power and sizeable storage space. RFID RW tag, based on those advantages, function to adjust and thus edit the RF signals from the labeling layers, resulting in application systems delivered by means of the Internet which works to cope with accordingly.

\section{System of EPC storage \& RFID Control-Mark}

This particular system, consisting of EPC storage \& RFID control marker, is highly involved with the backend database system, which operates on some certain hardware, linking and connecting relevant RFID tag information.

\section{Operating and Running principle}

\section{Savant Server}

Savant server, the specific core component of conveying the information within the EPC system with the aid of RW tag, functions to recognize and distinguish the information in the layer of electronic tag. Subsequently, this information is dispatch to cyber dispensation .

\section{ONS}

The ONS works to interpret the EPC code into the particular IP address, so as to identify the venue of the corresponding computers, and by achieving this, the ONS conducts relevant services

PML

PML functions with the following steps: first, real information is put into the database for storage ; this category of information includes ABI (auto basic information), OI(owner's information), as well as the instant traffic information. Second, PML is designed to solve the limited storage capacity problem by means of PML language. Third, by visiting the corresponding PML server, the electronic product coding becomes accessible to relevant real information operating procedure in that RW tag discriminates EPC from electronic tag and Savant Sever filters it, passing the messages onto ONS. Finally, PML after searching and obtaining the relevant IP address, acquires the information with it. The theoretical framework can be illustrated by Figure. 1 as follows.

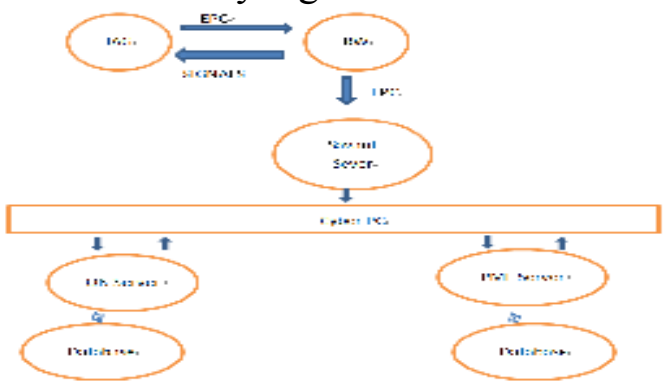

Figure.1. The theoretical framework 


\section{System \& Model Designs}

\section{Structures}

Due to the complexity extent and real-time characters of Canton's urban traffic network, application of intelligent control system has become a "must". In this structure, Canton's urban road traffic control system is designed to be categorized, accordingly, into point, line, surface, namely single intersection, the traffic trunk lines, together with regional network, working as a whole with controlling function. Intelligent control system is intended to separate the large system into micro-section sub-systems, regional system and macro-management intelligence system in traffic control center. They are decently designed for communicating, coordinating with each other in an effective and efficient manner. The whole system can be demonstrated as follows: micro sub-section system operates to update data of single section instantly ; signal timing acquires and applies those data ; regional intelligence system works to coordinate to reach the dynamic balance of road traffic flow and this process can be done by the analysis of regional traffic information.

\section{Working Process}

The working process can be summarized as follows. The upstream of intersection traffic lights is set and installed with a reader for transmitting signals, where induction current occurs when the autos with EPC pass through this section.

The specific reader is intended to discriminate the signals from antenna, decoding EPC and calculating the frequency of autos, followed the process of conveying the detected data to the reporting system. The data collected and thus acquired are applied by means of relevant updating and signal timing. Sub-section system operates to adjust the balance among roads by analyzing the information of traffic flow; Macro- management system coordinates all those flows.

The system, by combining those above mentioned, operated to conduct decision of adapting to the lights period, with the means of feedback information

\section{Control}

There exists a control procedure for this design in that signal period adjusts in a positive proportional manner with the single intersection traffic demand. Specifically, $\mathrm{T}$ is expected to act shorter as the single intersection traffic demand becomes fewer and fewer, nonetheless, the time generally should last longer than Px15s (P for phase digits), so as to avoid a certain phase of green time which lasts within $15 \mathrm{~s}$, by means of which autos can achieve a safety level when they going through crossroads. On the contrary, when the traffic demand grows bigger, the signal period $\mathrm{T}$ adjusts , accordingly, to serve a longer period, but generally within a 120 s period, or else some red light time period would turn to last over $60 \mathrm{~s}$, which has been proved beyond the drivers' patience. As long as the traffic demand comes to an extremely huge extent, the cycle control reaches a maximum degree. The process is illustrated by Figure.2.
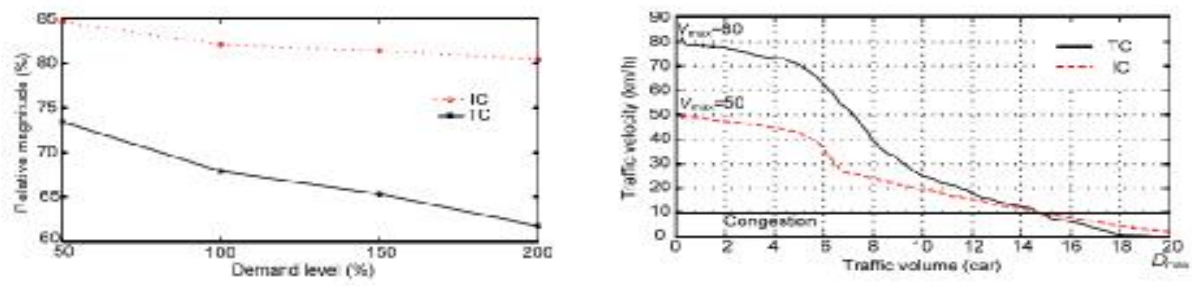

Figure.2. Demand level and Traffic Volume structure

The maximum $\mathrm{T}$ should be conducted when traffic congestion seems to be highly inevitable when the traffic demand reaches the peak. Intelligent traffic control system structure can be illustrated by the following diagram Figure. 3 


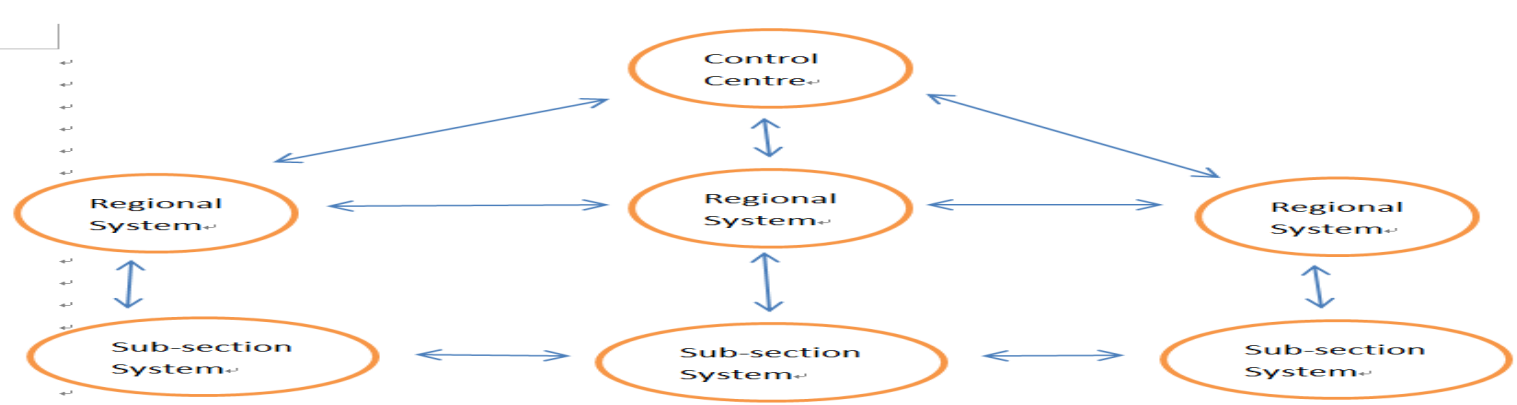

Figure.3 Intelligent traffic control system structure

\section{Conclusion}

On the basis of CDA-based Data Innovation-driven, Intelligent Traffic Control System Model has pushed the intelligent transportation system of Canton mega-city to a high level and proved to be an effective solution.Intelligent Traffic Control System Model can improve the efficiency of using the road and greatly reducing the energy consumption, empirical statistical study has revealed that Intelligent Traffic Control System Model has made traffic congestion reduction by 58.35\%; Short-distance commute efficiency enhanced by $65 \%$ or so; meanwhile the existing road capacity of Canton has increased by $59.63 \%$.Based on the CDA-based Data Innovation-driven, our Intelligent Traffic Control System Model has combined and integrated IT techniques, cyber techniques and geography-identification information techniques into an intelligent control traffic system.

By means of allocating diverse colors and modes to diverse roads, which is based on the road conditions resolved from real-time autos information, the real-time road condition can be well recognized on a the platform of the controlling center. An Empirical Study has followed up to check the effect of this model by the case study of Canton City in transportation system. A positive statistical results have been achieved, confirming the positive effect of this model. The results has shown a high practicability and reliability in practical use. With the data acquired, collected and innovatively-driven by monitors installed and decoded in intersections and sub-sections, different road conditions are discriminated, calculated and shown in diverse modes on the controlling layer to allocate efficient paths/routes to avoid the traffic congestion; meanwhile this model renders a guideline for a traffic authority to make decisions on traffic control.

\section{Acknowledgements}

This work was financially supported by the 2016 Guangdong Province philosophical social science planning project (GD16XYJ30), 2016 Guangzhou College of Technology and Business "Quality Engineering" construction project (ZL20161226) and 2016 Northeast Normal University education curriculum research center vocational education English teaching reform practice research project (NNUP04).

\section{References}

[1]Zhang G W. Introduction of Intelligent Transport Systems Engineering [M] Beijing: Publishing house of electronics industries, 2003.

[2] Zhang Wei. Design of Intelligent traffic control system [J]. Computer Knowledge and Technology 2011(10)

[3] Lu L, Zhang Hong. Simulation of city short-time traffic flow forecasting [J].Computer Simulation, 2012

[4] Li Nan. Meaning, basis and path of traffic resource integration [J].Logistics Engineering and Management 2010(195) 